\title{
Metaplasia óssea do endométrio: relato de caso
}

\section{Endometrium osseous metaplasia: a case report}

Álvaro Piazzetta Pinto1; Guilherme Barroso Guedes2; Felipe Francisco Bondan Tuon3

\begin{tabular}{l|l}
\multicolumn{1}{c|}{ Unitermos } & resumo \\
Endométrio & $\begin{array}{l}\text { Os autores relatam o caso de uma paciente que apresentou aborto seguido de infecção, resultando } \\
\text { em esterilidade secundária. Após curetagem uterina terapêutica, o laudo do exame anatomopatoló- } \\
\text { Infertilidade }\end{array}$ \\
Metaplasia óssea & $\begin{array}{l}\text { apresentou sinéquia de corpo e colo uterino. Apesar de rara, a metaplasia óssea endometrial deve ser } \\
\text { considerada no diagnóstico de infertilidade. O presente relato salienta a importância do diagnóstico } \\
\text { histopatológico e do conhecimento da etiopatogenia dessa entidade, sua associação com a endometrite } \\
\text { crônica e história de abortamento. }\end{array}$
\end{tabular}

abstract key words

The authors report a case of abortion, followed by infection with secondary infertility. After a uterine curettage, chronic endometritis with fibrosis, calcifications, and osseous metaplasia of the endometrium were detected by histopathology. In the follow up, the patient presented cervical and uterine synechia. Osseous metaplasia is a rare condition, but the clinician should think about it when he is in front of a patient with infertility. The present case report points out the importance of the histopathological diagnosis of osseous metaplasia, as well as the knowledge of its etiopathogenesis and association with chronic endometritis and abortion history.

Endometrium

Infertility

Osseous metaplasia

\section{Introdução}

Entre os fatores capazes de produzir esterilidade feminina, aqueles relacionados à mucosa endometrial raramente são mencionados. Apenas doenças inflamatórias, pólipos, presença de aderências, como na síndrome de Asherman, e processos destrutivos têm sido descritos na literatura. A metaplasia óssea é uma causa rara de infertilidade ${ }^{(2)}$. Essa condição parece ocorrer a partir da transformação do tecido conjuntivo mesenquimal em tecido ósseo, como resposta inflamatória e reparatória a um aborto prévio(1). O aborto espontâneo ou terapêutico é a principal condição predisponente para essa lesão, sendo que o tempo desde a agressão até o desenvolvimento completo da ossificação pode variar de oito semanas a $14 \operatorname{anos}^{(3)}$. Além da infertilidade secundária, a ossificação endometrial pode causar irregularidade menstrual e dismenorréia ${ }^{(6)}$. O objetivo deste relato de caso é descrever as características microscópicas dessa forma rara de infertilidade e revisar os aspectos ligados à etiopatogenia, tratamento e prognóstico da metaplasia óssea endometrial.

1. Professor-adjunto e ginecopatologista do Serviço de Anatomia Patológica do Hospital de Clínicas da Universidade Federal do Paraná (HC-UFPR); doutor em Patologia pela Faculdade de Medicina de São Paulo-USP.

2. Acadêmico de medicina do $10^{\circ}$ semestre da Universidade Federal do Paraná; bolsista do Programa Institucional de Bolsas de Iniciação Científica/Conselho Nacional de Desenvolvimento Científico e Tecnológico (PIBIC/CNPq).

3. Médico-residente de Clínica Médica do Hospital Universitário Evangélico de Curitiba (HUEC).

Trabalho realizado no Serviço de Anatomia Patológica do HC.UFPR. 


\section{Relato do caso}

Paciente feminina, 29 anos, G II; P I; A I, com queixa de infertilidade. Na história, refere aborto há dois anos seguido de infecção, o que resultou em esterilidade secundária. A paciente foi submetida à curetagem uterina única. $\mathrm{Na}$ análise anatomopatológica do material coletado, observou-se endométrio com glândulas estreitas, regulares, e revestidas por epitélio cilíndrico simples. No estroma foram verificadas extensas áreas de fibrose, com infiltração inflamatória monomorfonuclear e exsudação polimorfonuclear, em meio a focos de calcificação e ossificação, formando trabéculas ósseas imaturas, entremeadas por medula óssea. O diagnóstico anatomopatológico emitido foi de endometrite crônica com fibrose, calcificação e metaplasia óssea (MO). Na evolução, a paciente apresentou sinéquia de corpo e colo uterino.

\section{Discussão}

O primeiro relato da presença de tecido ósseo em mucosa endometrial ocorreu no ano de 1901, por um patologista alemão, e mais tarde alguns casos foram descritos esporadicamente. Em 1923, Thaler descreveu pela primeira vez a associação entre a ossificação endometrial e uma história prévia de aborto ${ }^{(10)}$.

Atualmente, sabe-se que aborto prévio, trauma póscuretagem e infecções do endométrio podem levar à metaplasia escamosa do epitélio glandular endometrial, seguida do aparecimento de cartilagem e finalmente tecido ósseo, sendo o processo denominado metaplasia óssea (MO).

Metaplasia é, por definição, a substituição de um tipo celular diferenciado por outro, também diferenciado. A explicação para o mecanismo de formação da $\mathrm{MO}$ baseiase no achado concomitante de epitélio escamoso e/ou porções de cartilagem em amostras de mucosa endometrial contendo focos de ossificação(9). Os fatores responsáveis pela estimulação do processo de $\mathrm{MO}$ endometrial ainda não estão bem esclarecidos.

Ganem et al. (1962) propuseram a hipótese da estimulação contínua por estrogênios, como fator determinante do processo ${ }^{(9)}$. Já Robinson (1964) sugeriu que a MO estava associada à calcificação distrófica da mucosa endometrial, ocasionada pela presença de tecido necrótico retido após aborto ${ }^{(7)}$. Em 1966, Roth e Taylor demonstraram a presença de mucopolissacarídeos em casos de MO do endométrio, sugerindo que o processo seria proveniente da formação de cartilagem em resposta à inflamação crônica e/ou trauma ${ }^{(8)}$.

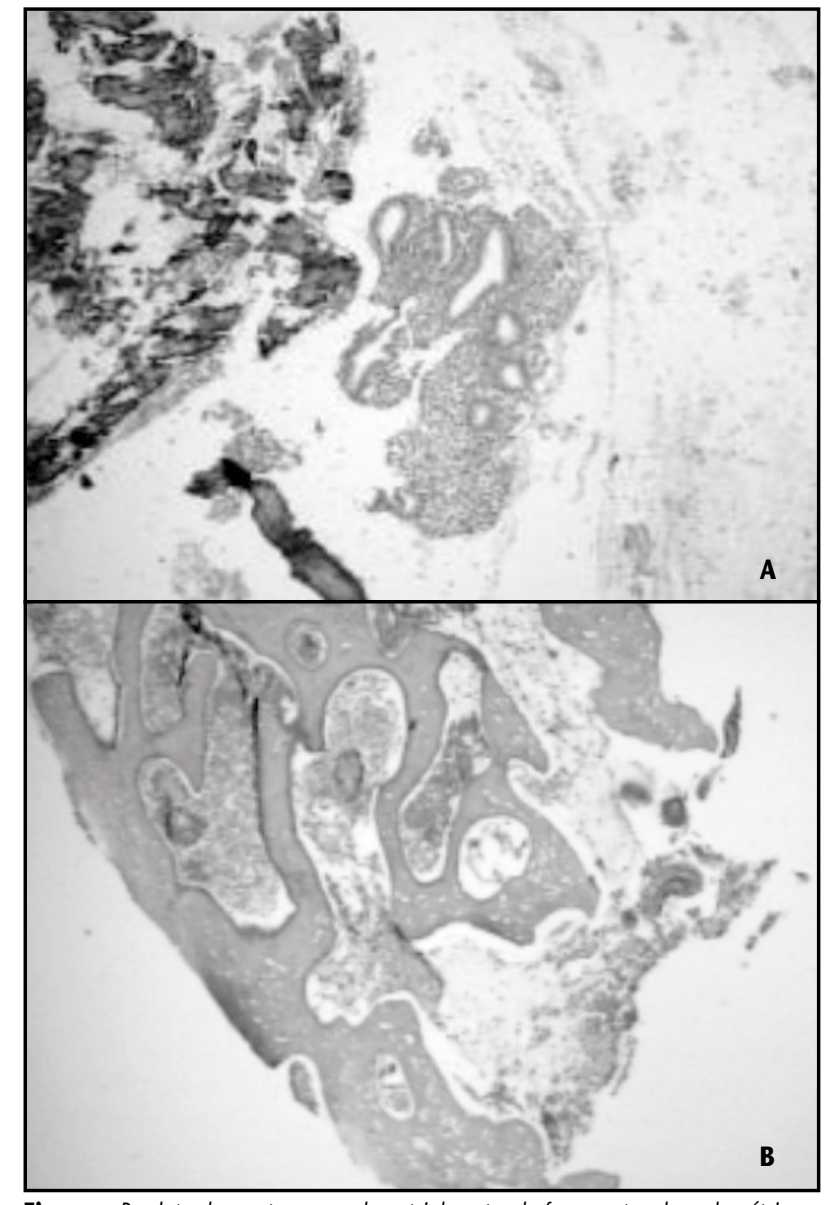

Figura - Produto de curetagem endometrial contendo fragmentos de endométrio alterado por processos de calcificação $(A)$ e metaplasia óssea (B)

Sugino et al. ${ }^{(9)}$ introduziram o conceito de que o sistema superóxido dismutase teria importante função no desenvolvimento da MO. Segundo esses autores, a inflamação crônica pós-aborto, a partir de restos teciduais retidos, poderia causar a liberação de fatores como radicais superóxido ou fator de necrose tumoral dos macrófagos, que por sua vez promoveriam a degeneração da mucosa endometrial. Essa agressão prolongada causaria então a transformação das células multipotentes do estroma em osteoblastos ${ }^{(1,6)}$.

A principal conseqüência da presença de tecido ósseo nas paredes uterinas é a infertilidade. Segundo Mayer (1901) o tecido ósseo agiria como um dispositivo intrauterino (DIU), provocando alterações locais que impediriam a fertilização(4).

Mais recentemente, Marcus et al. (1991) propuseram que a produção aumentada de prostaglandinas, devido à presença da MO, seria responsável pela infertilidade ${ }^{(5)}$.

No caso em questão, a etiologia do processo de MO parece estar relacionada à história de abortamento seguido de infecção. Corrobora com essa hipótese a observação de 
fibrose e inflamação crônica na amostra histopatológica, que levaram ao diagnóstico de endometrite crônica. Assim, ao patologista cabe estar atento para essa possibilidade, bem como para a distinção histológica entre os processos de calcificação e MO ilustrados (A e B) na Figura.

O prognóstico da MO quando manejada adequadamente é bom. Geralmente as pacientes retornam à fertilidade em um ano(1). A curetagem e a excisão orientada pela histeroscopia são as principais opções de tratamento ${ }^{(1,6,10)}$.

No caso relatado, a paciente foi submetida à curetagem única e evoluiu com sinéquia uterina e infertilidade irreversível. O presente estudo nos chama a atenção para o fato de que a remoção completa única pode levar a conseqüências indesejáveis, como as verificadas neste caso e as apontadas anteriormente também por outros autores.

\section{Referências}

I. ACHARYA, U.; PINION, S. B.; PARKIN, D. E. Osseous metaplasia of the endometrium treated by hysteroscopic resection. $\mathrm{Br}$ J Obstet Gynecol, v. 100, p. 391-2, 1993.

2. BAHCECl, M.; DEMIREL, L. C. Osseous metaplasia of the endometrium: a rare cause of infertility and hysteroscopy management. Human Reprod, v. I I, p. 2537-9, 1996.

3. BATHIA, N. N.; HOSHIKO, M. G. Uterine osseous metaplasia. Obstet Gynecol, v. 60, p. 2569, 1982

4. GANEM, K. J.; PARSONS, L.; FRIEDELL, G. H. Endometrial ossification. Am J Obstet Gynecol, v. 83, p. 1592-4, 1962.

5. MARCUS, S. F.; BAHATTACHARYA, J.; WLLIANS, G. et al. Endometrial ossification: a cause of secondary infertility. Am J Obstet Gynecol, v. 170, p. I381-3, 1991.
6. MAYER R. Knochegnewene im fotalen uterus. Z Geburtshilfe Gynekol, v. 46, p. 490-2, 1901.

7. ROBINSON, C. R. Endometrial ossification following a recent abortion. Can Med Assoc J, v. 90, p. 1317-8, 1964.

8. ROTH, E.; TAYLOR, H. B. Heteretopic cartilage in the uterus. Obstet Gynecol, v. 27,p. 838-44, 1966.

9. SUGINO, N.; SHIMAMURA, K.;AKIGUCHI, S. et al. Changes in activity of superoxide dismutase in the human endometrium throughout the menstrual cycle in early pregnancy. Hum Reprod, v. II, p. 1073-8, 1996.

10. THALER, H. Uberlebendes fotales knorpelgewebe im der uterushohel nach abortus. Zentalbl Gynakol, v. 46, p. I7847, 1923.

\section{Endereço para correspondência}

Dr. Álvaro Piazzetta Pinto Departamento de Patologia Hospital de Clínicas da Universidade Federal do Paraná Rua General Carneiro 181

CEP: $80069-900$ - Curitiba-PR

Telefone: (41) 2247988

Fax: (41) 3244684

e-mail: alvaropi@bsi.com.br 